10. Meyrick B, Christman B, Jesmok G. Effects of recombinant tumor necrosis factor-alpha on cultured pulmonary artery and lung microvascular endothelial monolayers. Am J Pathol. 1991;138:93-101.

Address for correspondence: Gilles Meyer, UMR1225, Ecole Nationale Vétérinaire de Toulouse, 23 chemin des Capelles BP87614, Toulouse CEDEX 3, France; email: g.meyer@ envt.fr

\section{Spotted Fever Group Rickettsia sp. Closely Related to R. japonica, Thailand}

To the Editor: In response to a recent report that suggested human infection with Rickettsia japonica in northeastern Thailand (1), we phylogenetically reexamined spotted fever group rickettsiae (SFGR) from Thailand. The organism had been isolated from a male Haemaphysalis hystricis tick found on Mt. Doi Suthep, Chiang Mai, northern Thailand, in December 2001. The strain was designated TCM1 and was not distinguishable from $R$. japonica by indirect immunoperoxidase stain using monoclonal antibody (2).

After propagating strain TCM1 in L-929 cell culture, we extracted DNA by using a Wizard Genomic DNA Purification Kit (Promega, Madison, WI, USA). We subjected the DNA to sequencing that targeted a 491-bp fragment of rickettsial outer membrane protein A (ompA), a 394-bp fragment of the rickettsial genus-specific 17$\mathrm{kDa}$ antigen gene, and a 1,250-bp fragment of citrate synthase gene (gltA). Direct sequencing of amplicons was performed as previously described. (3). Phylogenetic analyses based on
ompA indicated that strain TCM1 was closely related to and clustered within the same clade as $R$. japonica strain $\mathrm{YH}$ (98.4\% identity) (Figure, panel A). Also, a 17-kDa antigen gene obtained from strain TCM1 showed 99.5\% identity to the corresponding gene of $R$. japonica (Figure, panel B). Our phylogenetic analysis with ompA and 17-kDa antigen gene showed that strain TCM1 was closely related to $R$. japonica but distinguished from Rickettsia sp. PMK94 (which was closely related to $R$. heilongjiangensis from northeastern China) (3); another SFGR

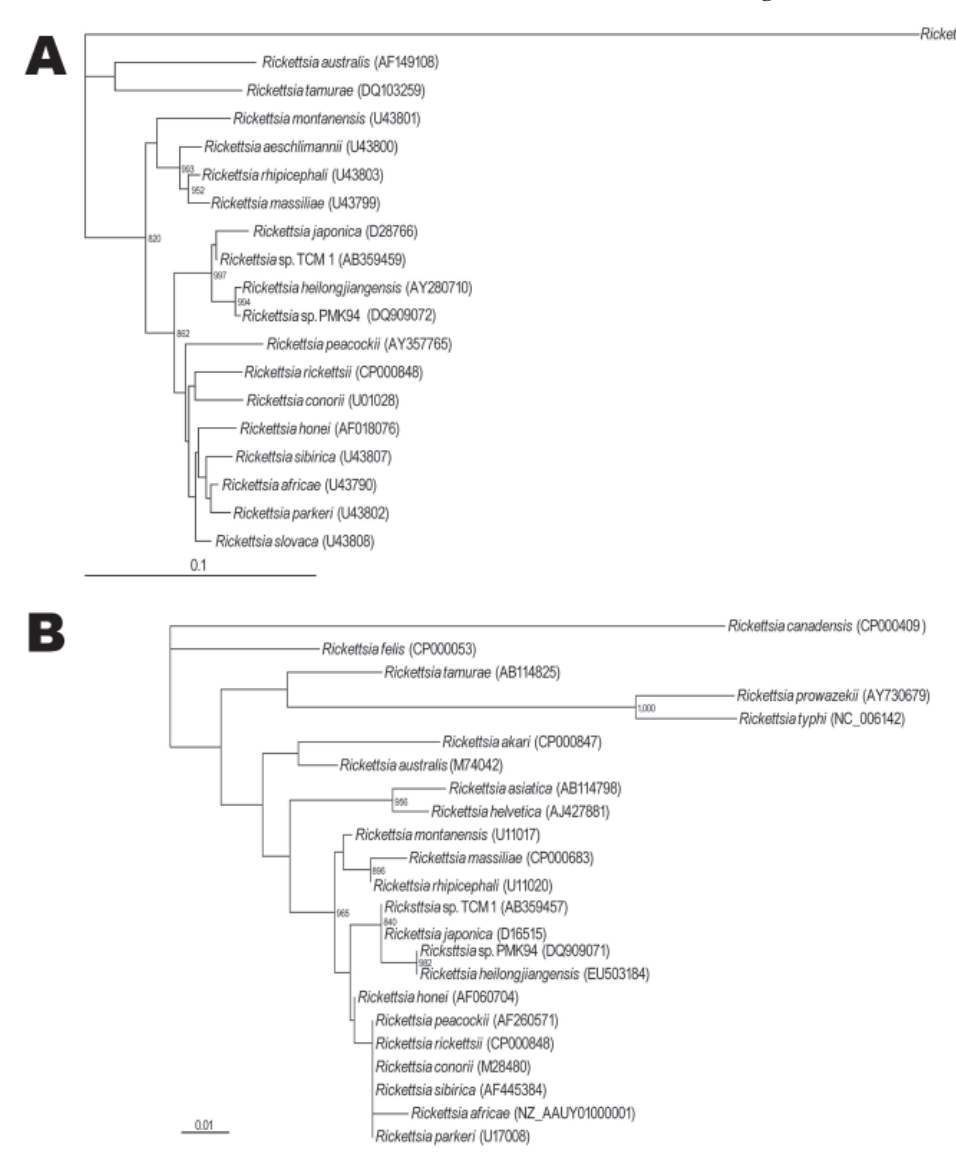

Figure. Phylogenetic analysis based on ompA gene (A) and rickettsial genus-specific 17-kDa antigen gene (B). Sequences were aligned by using the ClustalW software package (http:// clustalw.ddbj.nig.ac.jp/top-j.html), and neighbor-joining phylogenetic tree construction and bootstrap analysis were conducted according to the Kimura 2-parameter method (www. ddbj.nig.ac.jp). Pairwise alignments were performed with an open-gap penalty of 10 , a gap extension penalty of 0.5 , and a gap distance of 8 . Multiple alignments were also performed with the same values, and the phylogenetic branches were supported by bootstrap analysis with 1,000 replications (>800 were indicated). Rickettsia felis (CP000053) and $R$. canadensis (CP000409) were used as outgroups for ompA and 17-kDa antigen gene, respectively. The phylogenetic tree was constructed by using TreeView software version 1.5 (http://taxonomy.zoology.gla.ac.uk/rod/treeview.html). Scale bars indicate nucleotide substitutions (\%) per site. 
$R$. japonica is the specific pathogen of Japanese spotted fever, which has been found mainly in southwestern Japan (5). The present strain, closely related to $R$. japonica, is likely to have been isolated from $H$. hystricis in Thailand because $R$. japonica frequently has been isolated, or detected by PCR, from the same tick species in Japan (6). Such tick species-specificity of SFGR should be considered when speculating on any geopathologic relationships of rickettsioses among different SFGR-endemic areas. Previous reports on spotted fever-positive results of human serosurveys $(7,8)$ and on a clinical case (9) in northern Thailand may provide epidemiologic background. In Asia, multiple species of rickettsiea (e.g., $R$. japonica, $R$. heilongjiangensis, $R$. honei) are the causative agents of spotted fever rickettsioses, so the agent closely related to $R$. japonica could cause spotted fever in Thailand. Additionally, R. japonica has been found in Korea (10), and our current study indicates that $R$. japoni$\mathrm{Ca}$ and its genetic variants are widely distributed in Far Eastern countries, including Japan (Grant-in-Aid for International Cooperative Research, unpub. data). Therefore, the epidemiology and genetic variation of SFGR throughout Asia should be examined by molecular studies.

This work was supported in part by Grant-in-Aid for International Cooperative Research (nos. 13576005 and 19406008) from the Japan Society for the Promotion of Science.

\section{Nobuhiro Takada, Hiromi Fujita, Hiroki Kawabata, Shuji Ando, Akiko Sakata, Ai Takano, and Udom Chaithong}

Author affiliations: University of Fukui, Fukui, Japan (N. Takada); Ohara General Hospital, Fukushima, Japan (H. Fujita); National Institute of Infectious Diseases, Tokyo, Japan (H. Kawabata, S. Ando, A. Sakata, A. Takano); and Faculty of Medi- cine, Chiang Mai University, Chiang Mai, Thailand (U. Chaithong)

DOI: 10.3201/eid1504.081271

\section{References}

1. Gaywee J, Sunyakumthorn P, Rodkvamtook W, Ruang-areerate T, Mason CJ, Sirisopana N. Human infection with Rickettsia sp. related to $R$. japonica, Thailand. Emerg Infect Dis. 2007;13:671-3.

2. Fujita H, Takada N, Chaithong U. Preliminary report of rickettsial strains of spotted fever group isolated from ticks of China, Nepal and Thailand. Annual Report of Ohara General Hospital. 2002;44:15-8.

3. Zhang JZ, Fan MY, Wu YM, Fournier PE, Roux V, Raoult D. Genetic classification of "Rickettsia heilongjiangii" and "Rickettsia hulinii," two Chinese spotted fever group rickettsiae. J Clin Microbiol. 2000;38:3498-501.

4. Kollars TM Jr, Tippayachai B, Bodhidatta D. Short report: Thai tick typhus, Rickettsia honei, and a unique Rickettsia detected in Ixodes granulates (Ixodidae: Acari) from Thailand. Am J Trop Med Hyg. 2001;65:535-7.

5. Mahara F. Rickettsioses in Japan and the Far East. Ann NYAcad Sci. 2006;1078:60 73. DOI: 10.1196/annals.1374.007

6. Fujita H, Takada N. Diversity of genus Rickettsia detected from ticks in Japan. Acari and emerging/reemerging infectious diseases [in Japanese]. Tokyo: Zenkoku Noson Kyoiku Kyokai Publishing; 2007. p. 129-39.

7. Takada N, Fujita H, Yano Y, Huang W-H, Khamboonruang C. Serosurveys of spotted fever and murine typhus in local residents of Taiwan and Thailand compared with Japan. Southeast Asian J Trop Med Public Health. 1993;24:354-6.

8. Parola P, Miller RS, McDaniel P, Telford SR, Rolain J-M, Wongsrichanalai C, et al. Emerging rickettsioses of the Thai-Myanmar border. Emerg Infect Dis. 2003;9:592-5.

9. Sirisanthana T, Pinyopornpanit V, Sirisanthana V, Strickman D, Kelly DJ, Dasch GA.. First cases of spotted fever group rickettsiosis in Thailand. Am J Trop Med Hyg. 1994;50:682-6.

10. Lee J-H, Park H-S, Jung K-D, Jang W-J, Koh S-E, Kang S-S, et al. Identification of the spotted fever group rickettsiae detected from Haemaphysalis longicornis in Korea. Microbiol Immunol. 2003;47:301-4.

Address for correspondence: Nobuhiro Takada, University of Fukui, Faculty of Medical Sciences 23, Matsuoka, Eiheiji Fukui 910-1193, Japan; email: acari@u-fukui.ac.jp

\section{Segniliparus rugosus Infection, Australia}

To the Editor: Recently, a female teenager with cystic fibrosis who resided in tropical north Queensland, Australia, was found to be infected with Segniliparus rugosus. She was homozygous for the deltaF508 mutation, had well-preserved lung function, and regularly played competitive sports. Unlike many cystic fibrosis patients, she did not have a history of chronic Pseudomonas aeruginosa infections, but Stenotrophomonas maltophilia and Achromobacter xylosoxidans had been previously isolated from her sputum. In May 2007, she described reduced exercise tolerance and increased cough with excess sputum production. Lung function testing showed modest spirometric decline. A computed tomographic scan of the chest showed significant mucus plugging and bronchiectasis, uncommon without previous $P$. aeruginosa infection. Sputum was $3+$ smear positive for acid-fast bacilli (AFB), and S. rugosus was isolated from liquid culture. Empiric antimicrobial drug therapy was changed to rifabutin and co-trimoxazole because these drugs have been effective in previous cases (1). Clinically, the patient showed response to the treatment. After 12 months of treatment, her sputum was still $3+$ positive for AFB, and S. rugosus was again found in culture. She was referred to a pediatric teaching hospital in Brisbane with worsening respiratory symptoms precipitated by influenza B infection. Antimicrobial drug therapy with intravenous imipenem, oral moxifloxacin, and co-trimoxazole for 2 weeks resulted in clinical improvement but little reduction in smear positivity.

The initial AFB smear-positive sputum specimen underwent routine decontamination with sodium hydroxide and neutralization and was inoculated into radiometric 12B vials (Bec- 